\title{
Designing and Testing a New Shack-Hartmann High Bandwidth Two-Dimensional Wave front Sensor
}

\author{
Shaddy Abado ${ }^{*}$, Stanislav Gordeyev ${ }^{\dagger}$, and Eric J. Jumper ${ }^{*}$ \\ Center for flow physics and control \\ University of Notre Dame, Notre Dame, IN 46656
}

\begin{abstract}
A novel Shack-Hartmann high bandwidth two-dimensional wave front sensor was designed and tested, addressing high temporal bandwidth light aberrations due to light-fluid interaction. The new sensor's principles of operation and modifications are presented, and compared against a commercially-available two-dimensional wave front sensor with a $33 \times 44$ sub-apertures spatial resolution. The comparison was performed over a two-dimensional, acoustically forced heated jet. The results show a good agreement between the two sensors.
\end{abstract}

\section{Nomenclature}

$\begin{array}{ll}D & =\text { jet exit nozzle width } \\ \text { DivX } & =\text { light spot position in } x \text { direction } \\ \text { DivY } & =\text { light spot position in } y \text { direction } \\ M & =\text { telescope magnification } \\ O P D & =\text { optical path difference } \\ O P D_{p-p} & =\text { peak to peak of optical path difference } \\ O P D_{r m s} & =\text { root-mean-square of optical path difference } \\ O P L & =\text { optical path length } \\ S_{x}, S_{y} & =\text { Instantaneous phase gradients } \\ U_{c} & =\text { convection velocity } \\ f & =\text { focal length } \\ n & =\text { index-of-refraction } \\ s & =\text { beam path } \\ t & =\text { time } \\ x, X & =\text { streamwise direction } \\ y, Y & =\text { spanwise direction } \\ Z, Z & =\text { cross-stream direction } \\ \theta & =\text { beam deflection angle } \\ \epsilon & =\text { spot displacement } \\ \lambda & =\text { wavelength } \\ \Phi & =\text { phase error }\end{array}$

\section{Introduction}

$\mathrm{W}$ HEN a perfectly collimated, planar beam of light propagates through a field with variable index-of-refraction it gets distorted, causing a significant reduction in the optical signal intensity in the far field, and degradation in overall light transmitting system performance. When the transmitted system is airborne these effects are called aero-optical effects ${ }^{1}$.

\footnotetext{
${ }^{*}$ Graduate research assistant, Department of Aerospace and Mech. Eng, AIAA Student Member.

${ }^{\dagger}$ Assistant Research Professor, Department of Aerospace and Mech. Eng., Member AIAA.

* Professor, Department of Aerospace and Mech. Eng., Fellow AIAA.
} 
Contrary to atmospheric optical aberrations, which are caused by temperature gradients in the far field of the beam exit pupil ${ }^{2}$, aero-optical active aberration area is in the near field and caused by density gradients due to boundary layers, separated shear layers, and waves which are characterized with high temporal frequencies aberations ${ }^{3}$, typically around several kilohertzs, see Fig. 1.

To measure optical aberrations, compensate for the intensity loses, and maximize propagated beam energy on a target, a closed loop adaptive optics system (AO) is typically used; such system senses the aberration, using a wave front sensor (WFS), reconstructs the wave front phase to estimate and predicate phase aberrations using a reconstructor, and constructs a conjugate correction using a deformable mirror $(\mathrm{DM})^{4}$.

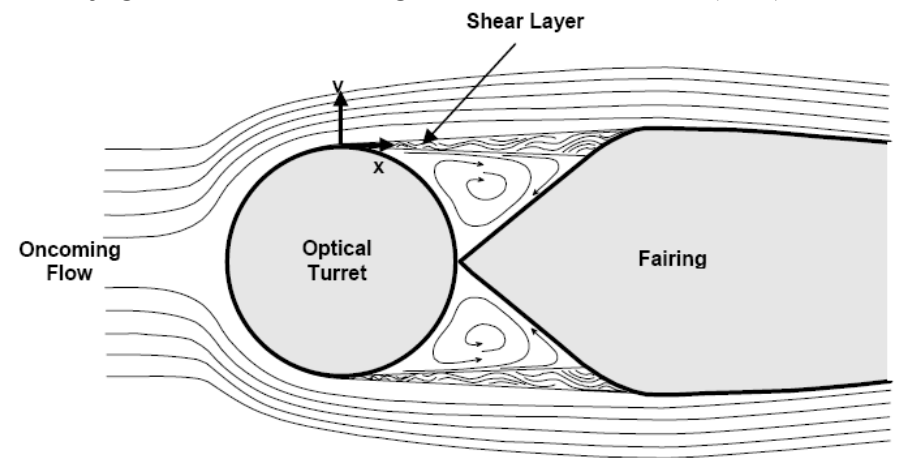

Figure 1. Flow over a turret/fairing combination.

It was shown by Nightingale et al. ${ }^{3}$ that in order to achieve an adaptive optics system which is capable of operating in a real flight scenario, a wave front sensor with a real time framing rate that is on the order of 100 times of the bandwidth of an aberration is required. As it was mentioned, aero-optical disturbances are characterized with high temporal frequencies around a several kilohertzs, hence a wave front sensor that operates in a real time sampling rate of hundreds of kilohertz is required. Conventional adaptive optics systems, which are limited to hundreds of hertz disturbances correction capability for the current state-of-art systems, cannot be used to compensate aero-optics intensity loses, and different approaches need to be considered to compensate for the aberration effects on the transmitted beam.

In addition, the wave front sensor should provide wave fronts with a minimum delay between sensing and calculating wave fronts for adaptive optics system to be stable ${ }^{3}$. High framing rate and fast wave front reconstruction are above the current capability of most of the commercially available, digital charge-coupled devices (CCD) wave front sensors; there is the need to design a high bandwidth wave front sensor.

With this in mind, a new 2-D high-bandwidth Shack-Hartmann wave front sensor (SHWFS) was designed, capable of acquiring data at greater than $100 \mathrm{kHz}$ temporal rate, in addition to a spatial resolution of one-tenth of the aperture. This novel sensor can be used to address high temporal bandwidth aero-optical aberrated flows that cannot be addressed by the current availably state-of-art wave front sensors. This sensor is a third generation of a sensors originally developed by Cicchiello, Hugo, Cheung, Jumper, Minniti, Preston and Cavalieri under a joint SBIR program between Notre Dame and Oceanit ${ }^{5}$.

The wave front sensor can be used in two major ways. The first is as part of a conventional real (or pseudo-real) time adaptive optics system capable of diagnosing and compensating for optical disturbances at high temporal rates, maintaining the system stability. Furthermore, the wave front sensor can be used as a diagnostic tool, investigating optical disturbances associated with turbulence flows, up to at present a maximum frequency of $52 \mathrm{kHz}$ limited by the Nyquist criterion; the current limit is due to the bandwidth limitation of our presently configured data acquisition system. Results of this capability were given in this paper.

Wave front aberrations are commonly quantified using optical path difference (OPD), which is the meanremoved optical path length (OPL). The $O P L$ is defined as the path integral of the index of refraction field, $n$, along the beam path, $s$,

$$
O P L(t, x, y)=\int n(t, x, y) d s
$$

$O P D$ is obtained by removing the mean over the aperture,

$$
O P D(t, x, y)=O P L(t, x, y)-\overline{O P L(t)}
$$


Spatial $O P D$ root-mean-squares $\left(O P D_{r m s}\right)$ and OPD peak-to-peak $\left(O P D_{p-p}\right)$ values are typically calculated to quantify the deviation from a perfectly collimated beam.

To verify the validity and accuracy of the new designed wave front sensor, a test benchmark experiment was conducted over a heated jet facility at University of Notre Dame Hessert Laboratory. A low-speed commercially available 2-D wave front sensor was used to make benchmark measurements. Streamwise evaluations of roll-up structures were correctly captured in both low and high-speed wave front sensors.

Section 2 of the paper will present a detailed description of Shack-Hartmann wave front sensor method of operation, and the modification for the conventional sensor to improve the sensor's accuracy, sensitivity and sampling rate. A wave front phase reconstruction algorithm, that was developed for the new sensor layout configuration, will be derived and presented in Section 3. A brief description of the commercially available wave front sensor and the heated jet facility are presented in the last part of Section 3 and in Section 4. A description of the test setup and investigation of the baseline flow are discussed in Section 5. In addition, wave front sensors comparison between the new designed wave front sensor and the commercially available one will be discussed. The paper concludes with a discussion of the presented results and future work.

\section{Wave front Sensors}

\section{A. Shack-Hartmann Wave front sensor}

In the early 1900s Hartmann proposed a sensor to measure wave front aberrations by measuring the transverse ray aberration in the image plane ${ }^{6}$. Based on the Huygen's principle, a ray of light travels normal to its associated optical wave front ${ }^{7}$, Hartmann placed a mask with holes in front of the incoming beam, and a photographic plate a known distance, $d$, from the mask. See in Fig. 2, left.

By measuring the displacement, $\epsilon$, of each light ray from its unaberrated position, and knowing the distance from the mask to the photographic plate, $d$, it is possible to find the local slope, $\theta_{x}$, and reconstruct the wave front phase by integration. The main disadvantage of Hartmann plate test is the requirement for a unique mask for each tested optics.

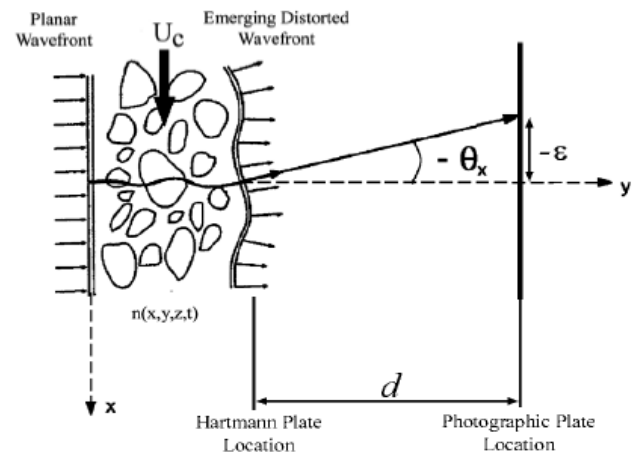

\section{Sample Photographic Plates}
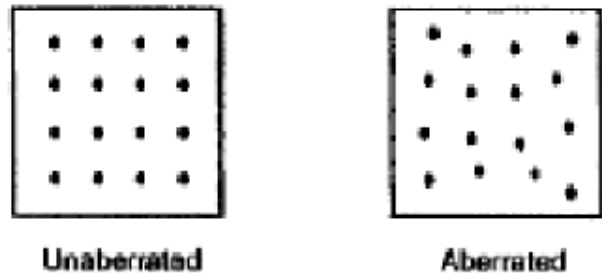

Figure 2. Hartmann plate test, left. Sample photographic plates for unaberrated and aberrated wave front, right.

The Shack-Hartmann wave front sensor ${ }^{4}$ is a modification of the classical Hartmann plate test. For this sensor, the mask was replaced by a 2-D lenslet array with a focal length, $f$, fixed in front of a position sensing device (PSD), such as CCD or photodiodes, instead of a photographic plate, as show in Fig. 3.The lenslet array spatially divides the incoming beam into an array of sub-apertures, where the deflection angle, $\theta$, over each sub-aperture represents the average wave front local tilt of the incoming beam, and independent of the intensity pattern across the sub-aperture. Each lenslet sub-aperture is associated with a PSD which evaluates the local transverse dot displacement offset, $\epsilon$, directly related to the local wave front gradient, $\theta$, of the wave front by equation

$$
\theta \approx \frac{\epsilon}{f}=\nabla(O P L)
$$

The wave front is uniquely reconstructed by knowing the slopes at an array of finite points using either direct or iterative integration methods to solve Eq. (3) for the entire 2-D array ${ }^{12}$. The spatial resolution of the sensor 
determined by the lenslet focal length, $f$, and sub-apertures spacing. The sensor sensitivity, $\theta_{\min }$, is determined by the minimum detectable change in focal spot position, $\epsilon_{\min }$, based on the equation

$$
\theta_{\min }=\frac{\epsilon_{\min }}{f}
$$

hence, increasing the focal length, $f$, of the lenslet array will improve the measurement sensitivity. The temporal resolution is basically limited to the sampling rate of the detectors at which one is able to determine all the spots displacements. To permit sensor operation at high temporal bandwidth, we would ideally want to sample wave fronts at high resolution and minimize computation time.

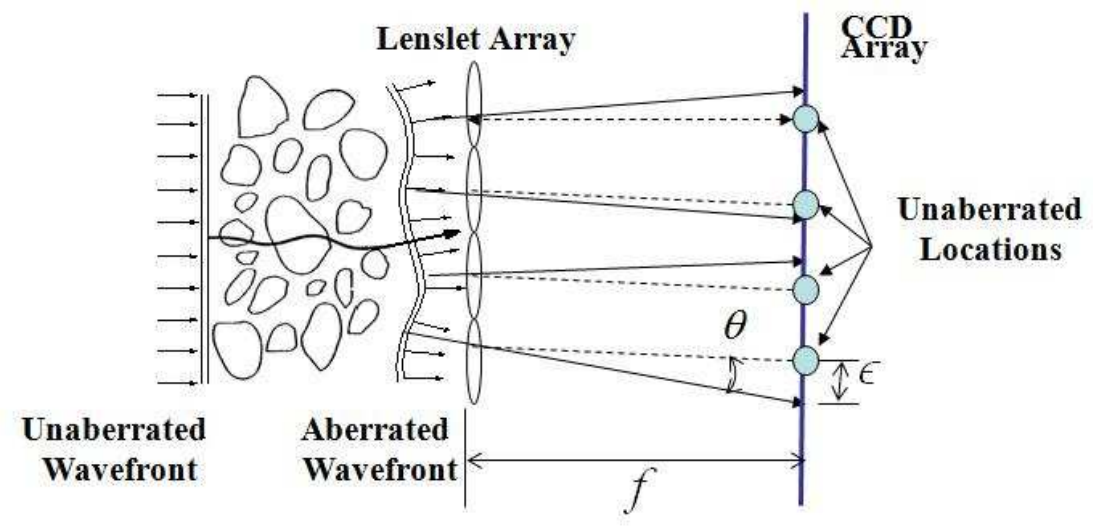

Figure 3. Shack-Hartmann wave front sensor: principle of operation.

\section{B. High-bandwidth wave front sensor}

As it was mentioned above, aero-optics effects are associated with high temporal frequencies exceeding the current framing capability of commercially available wave front sensors based on CCD detectors; such sensors grab the spots image and then use a data-reduction algorithm to find the spots positions. It should be mentioned that highframing rate CCD cameras do exist; however, at preset running these at high frequency reduces the pixel count available per sub-aperture. Theoretically at least four pixels are needed to resolve the focus spot centroid as a quad cell. Shack-Hartmann sensors using these high-bandwidth CCD arrays have been constructed for research demonstrations ${ }^{8}$; however, there are still complications in turning these into real-time sensors.

A number of wave front sensors were developed by the aero-optics group in Notre Dame, the first was a 1-D SABT (Small-Aperture Beam Technique) wave front sensor introduced by Jumper et al. ${ }^{9}$ which demonstrated a wave front capturing rate up to $100 \mathrm{kHz}$. A second generation of $8 \mathrm{X} 8$ sub-apertures high-bandwidth ShackHartmann wave front sensor (SHWFS) was designed under a joint SBIR between Notre Dame and Oceaint ${ }^{5}$ referenced earlier, and tested ${ }^{10,11}$, a wave front capturing rate up to $125 \mathrm{kHz}$ was demonstrated for the $8 X 8$ subaperture wave front sensor.

The new designed high bandwidth wave front sensor has a 68 duo-lateral analog position sensing photodiodes with an active area of $4 \times 4 \mathrm{~mm}$ and maximum rise time of $1 \mu$ s from Pacific Silicon Sensor. The photodiodes converts an incident light spot into continuous position data, the generated currents are transferred into voltages by current-to-voltage transimpedance amplifiers, and seen as four output voltages, $V_{1}$ and $V_{2}$ for $x$ direction, and $V_{3}$ and $V_{4}$ for $y$ direction, the relation between the voltages gives the light spots positions through the formulas

$$
\text { Position } x=K_{x} \cdot \frac{V_{1}-V_{2}}{V_{1}+V_{2}} \text { and Position } y=K_{y} \cdot \frac{V_{3}-V_{4}}{V_{3}+V_{4}}
$$

where, $K_{x}$ and $K_{y}$ are calibration coefficients which are constant over a large area of the detector. The 68 detectors were mounted on a single substrate and arranged in a $85 \mathrm{~mm}$ diameter circular pattern $(10 \times 10$ with removed corners) and $9 \mathrm{~mm}$ space between each two adjacent PSDs, see Fig. 4, left. The 272 analog voltage signals are sampled by a three data acquisition boards from Microstar Laboratories, Inc., controlled by a data acquisition computer, also used to post-process the acquired data. The sensor electronic design was improved by designing a single transimpedance amplifiers board, which decreases the number of required connection wires, and reducing the 
electronic rack size significantly, compared to the previous $8 X 8$ wave front sensor ${ }^{10,11}$, were BNC cables were used to connect the system components and four transimpedance amplifiers breakout boxes were used, thus, simplifying the use of the system in different facilities. The new sensor with its electronics are shown in Fig. 4, left, a photo of the old electronic rack data acquisition system is shown in Fig. 4, right.
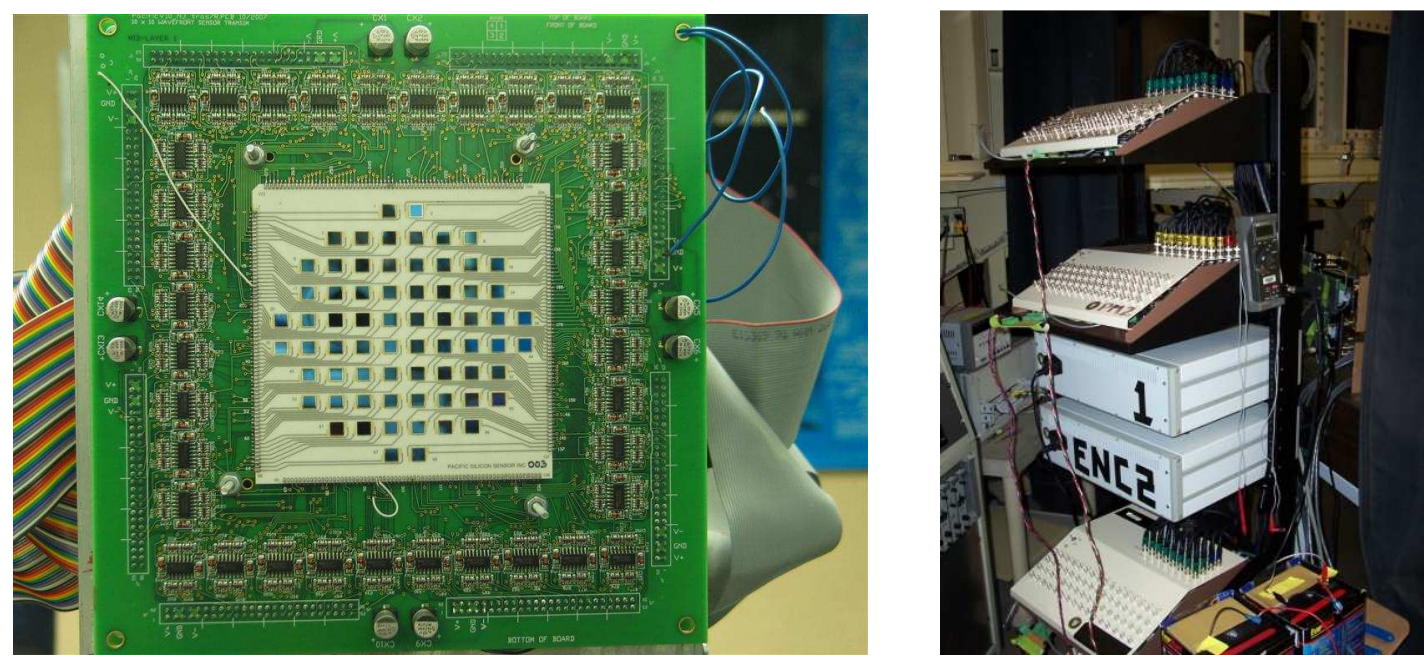

Figure 4. A Layout of 10x10 wave front sensor and its electronics, left. Old electronic rack, right.

Replacing the charge-coupled device (CCD) array with an array of analog PSDs offers an accurate spot position measurement, a high analog resolution, and a fast response time that could not be achieved with the commercially available wave front sensors. Therefore, the time-consuming CCD data-reduction algorithm was replaced by a simple spot centroid measurement, achieving a sampling frame rates greater than $100 \mathrm{kHz}$, defined only by the sampling speed of $\mathrm{A} / \mathrm{D}$ conversion boards.

One key requirement of a wave front sensor system is to have a optical bench size, and a maximized sensitivity. A monolithic lenslet array, with $55 \mathrm{~mm}$ focal length and $25 \mathrm{~mm}$ diameter size, from Adaptive Optics Associates, Inc. was used, reducing the lenslet array size significantly, compared to the previous generation of $8 X 8$ wave front sensor, where a custom made 4" lenslet array with a focal length of $0.5 \mathrm{~m}$ was used. To improve the sensor sensitivity, a re-imaging telescope was designed; the telescope uses three off-the-shelf lenses, two negative lenses and a positive one, to re-image the lenslet array original image plane onto the detector plane with $9 X$ magnification. The telescope was designed using optical designing software to avoid static aberrations and curvature of the field. The two negative lenses diverge the lenslet spots focal plane, while the positive one re-collimate, and re-image them to the detectors board, hence, the lenslet focus length is increased from $f$ to an effective focal length of $M \cdot f$. Figure 5, right, gives a schematic of the telescope optical layout. With the re-imaging telescope Eq. (4), describing the sensor sensitivity, can be re-written as,

$$
\theta_{\min }=\frac{\epsilon_{\min }}{M \cdot f}
$$

where, $M$ is the telescope magnification, $f$ is the lenslet focal length, and $\epsilon_{\min }$ is the minimum detectable focal spot displacement in the image plane of the wave front sensor due to the local aberrations in the wave front phase. Based on Eq. (7), aberrations that could not be detected without the re-imaging telescope due to the PSD physical limitation, could be magnified and detected. Fig. 5, left presents the designed telescope case, containing the lenslet array, and the three lenses. 

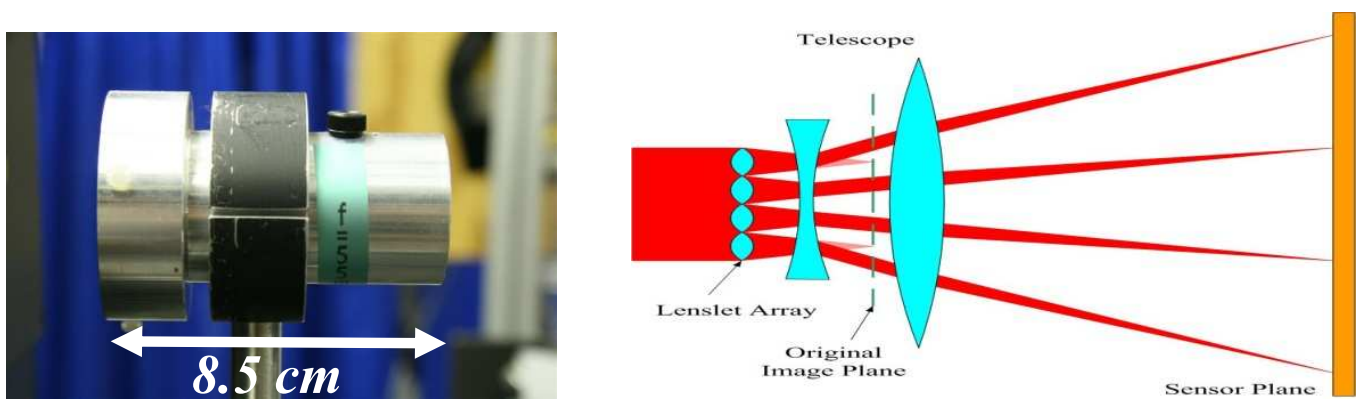

Figure 5. Designed re-imaging telescope, left. Telescope optical layout with light entering the lenslet from the left, right.

Each component of the new system: photodiodes detectors, detectors layout, electronics, and lenslet array reimaging telescope, introduced a significant improvement to the conventional Shack-Hartmann wave front sensor sensitivity, accuracy, size and sampling rate, compared to the previous generation of $8 X 8$ sub-apertures highbandwidth Shack-Hartmann wave front sensor. Images of the assembled wave front sensor case, and the electronic boards inside the case, are shown in Fig. 6.
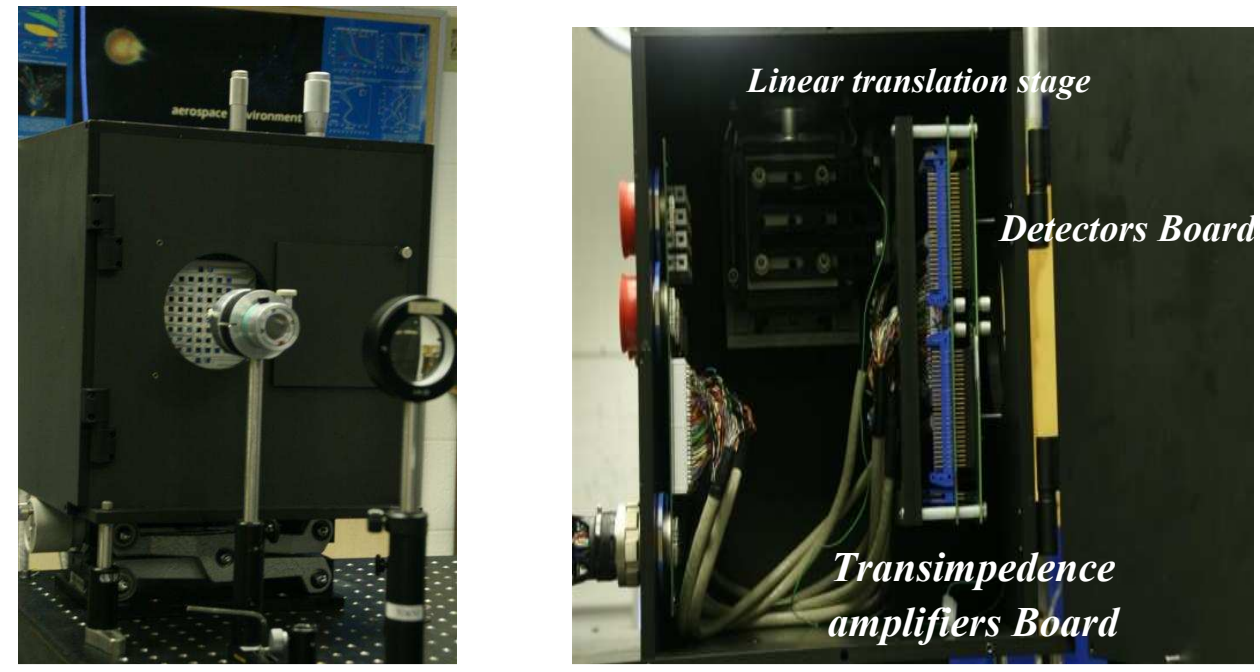

Figure 6. Wave front sensor case, containing the detectors board, transimpedance amplifiers board, and two dimensional linear translation stage, left. An image of the electronic boards inside the wave front sensor case, right.

\section{Commercially available Wavefront Sciences wave front sensor}

A Wavefront Sciences $C L A S-2 D$ wave front sensor was used as a benchmark sensor to evaluate and validate the new-design wave front sensor. The CLAS-2D sensor has a $33 X 44$ sub-apertures resolution, and uses a CCD array camera to detect spots displacement in the focal plane (cf. above). The camera is framed by an off-the-shelf frame grabber, which can be externally triggered to capture a single frame, or can be run at a frame rate up to $30 \mathrm{~Hz}$. Obviously, the sensor frame rate is insufficient to time resolve aero-optical flows. However, the present study is conducted over periodic aero-optical aberrations caused by an acoustically-forced, two-dimensional heated jet, as will be described later. Hence, the sensor can be used to collect a spatially-resolved phase-locked wave front for the purpose of comparison with the time resolved high bandwidth wave front sensor.

\section{Wave front Reconstruction Algorithm}

An essential part of a wave front sensor system is the wave front reconstruction algorithm, used to reconstruct and evaluate the instantaneous aberrated wave front phase. As previously mentioned, the Shack-Hartmann wave 
front sensor measures the wave front local phase gradients, $S_{x}$ and $S_{y}$, which are integrated to calculate the unknown wave front phase, $\Phi$. Eq. (3) can be re-written as

$$
\nabla \Phi(x, y)=\vec{S}(x, y)
$$

The incident wave front is described in terms of the Cartesian coordinates $(x, y)$.

For a real time AO system, the wave front reconstruction algorithm is integrated in the conjugate constructor (CC) processor, controlling the closed loop system. Hence the significance of reconstructing the wave front phase accurately and efficiently, reducing system latency and maintaining system stability.

Different solution methods have been developed by a number of authors ${ }^{12-14}$. For our analysis, Fried geometry ${ }^{12}$ was adapted, were each PSD was represented as a square sub-aperture (cell) with the measured orthogonal phase gradients in its center, and the four estimated phases (nodes) lie in the sub-aperture corners. Notating the elements by $(i, j)$ notation, each sub-aperture was represented as shown in Fig, 7, left. The grid array, shown in Fig. 7, right, was achieved when applying the geometrical discretization to the $10 X 10$ sensor layout. In our case, the grid array has 68 cells and 89 nodes.

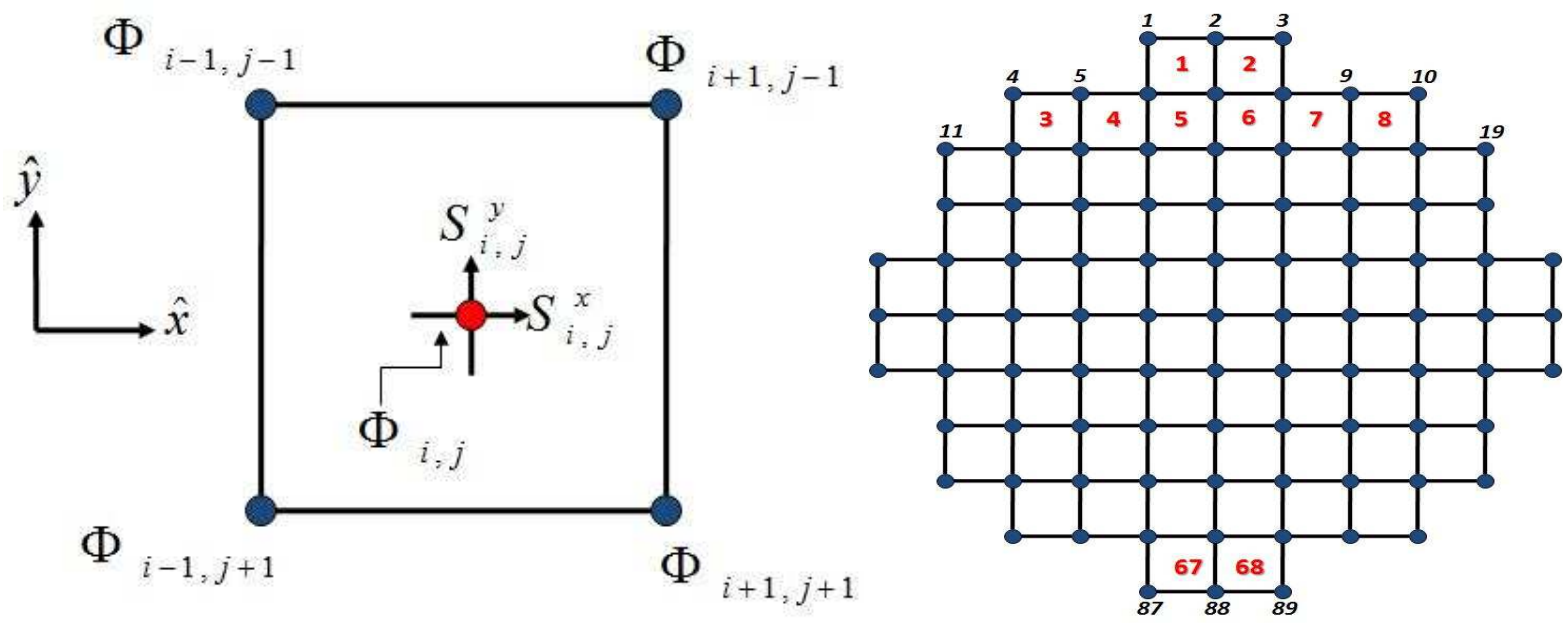

Figure 7. Fried geometry cell, the measured phase gradients are in the center and the estimated phases lie in the corners (nodes), left . Wave front sensor grid array with 68 cells and 89 nodes, right

Least-squares estimation was assumed to fit a bilinear curve solving Eq. (8). The relation between the phase and the phase gradients, given at Eq. (8), can be written as,

$$
\frac{\partial \Phi}{\partial x}=S_{x}, \frac{\partial \Phi}{\partial y}=S_{y}, \text { and } \quad \frac{\partial^{2} \Phi}{\partial x \partial y} \rightarrow 0
$$

Then, the least square estimation can be written as,

$$
\Delta=\left(\frac{\partial \Phi}{\partial x}-S_{x}\right)^{2}+\left(\frac{\partial \Phi}{\partial y}-S_{y}\right)^{2}+\left(\frac{\partial^{2} \Phi}{\partial x \partial y}-0\right)^{2} \rightarrow \text { Minimum }
$$

The third term is designed to suppress high spatial frequencies that can be found in each sub-aperture, and to make sure that Eq. (12) has a unique solution.

A second order central difference method can be used to discretize the partial derivative, and Eqs. (9)-(11) can be re-written using matrix notation as,

$$
A_{x} \Phi=S_{x} \quad, \quad A_{y} \Phi=S_{y} \quad \text {, and } \quad A_{x y} \Phi=0
$$


where, $A_{x}, A_{y}$, and $A_{x y}$ are matrix derivative operators $\frac{\partial \Phi}{\partial x}, \frac{\partial \Phi}{\partial y}$, and $\frac{\partial^{2} \Phi}{\partial x \partial y}$, respectively. The operators are rectangular sparse matrices with only 4 non-zero elements at each row. Each row or column of the matrix represents a different cell or a node, respectively. For the $10 x 10$ wave front sensor, the matrix dimensions are [68x89]. Using the matrix notation Eq. (12) can be re-written as,

$$
\Delta=\left(A_{x} \Phi-S_{x}\right)^{2}+\left(A_{y} \Phi-S_{y}\right)^{2}+\left(A_{x y}-0\right)^{2} \rightarrow \text { Minimum }
$$

Taking the derivative with respect to $\Phi$, and imposing a zero gradient on one of the nodes such that a unique solution can be found, Eq. (12) can be finally written as,

$$
\Phi=C_{x} S_{x}+C_{y} S_{y}
$$

where, the reconstruction matrices, $C_{x}=A^{-1} A_{x}^{T}$ and $C_{y}=A^{-1} A_{y}^{T}$, are independent of the input measured slopes, and are needed to be calculated once for the 10x10 sensor layout configuration and stored. If needed, the reconstruction matrices, $C_{x}$ and $C_{y}$ can be stored on a digital multiplication board integrated in a real time AO system.

\section{Two-Dimensional Heated Jet Facility}

\section{A. Facility description and jet response to acoustic forcing}

The experiments were conducted at the heated-jet facility at the University of Notre Dame. A detailed description of the facility and flow measurements can be found in Ref. 15-17. Here, we provide a short description only.

The heated-jet facility consists of a plenum chamber pressurized above atmospheric pressure by a squirrel-cagetype blower. After passing through filters and a slight contraction, air enters a rectangular duct with a series of heaters located approximately $1 \mathrm{~m}$ from its entrance and $1 \mathrm{~m}$ from a $90^{\circ}$ finned bend so that the heated air moves vertically in a rectangular duct with filters and flow straighteners, as shown in Fig. 8, left. The flow then passes through a 16-to-1, two-dimensional nozzle to an exit nozzle width, $D$, of $\frac{1}{2}$ ". Two end plates are aligned in the flow direction to help reduce three-dimensional effects that would otherwise be present if the jet were to be uncontained. The heated jet core velocity, $U_{c}$, is approximately $7 \mathrm{~m} / \mathrm{s}$. A side and top view of the facility are shown in Fig. 8 , left. A front and side view of the jet nozzle exit plane, in addition to a number of main geometrical dimensions are shown at Fig. 8, right.

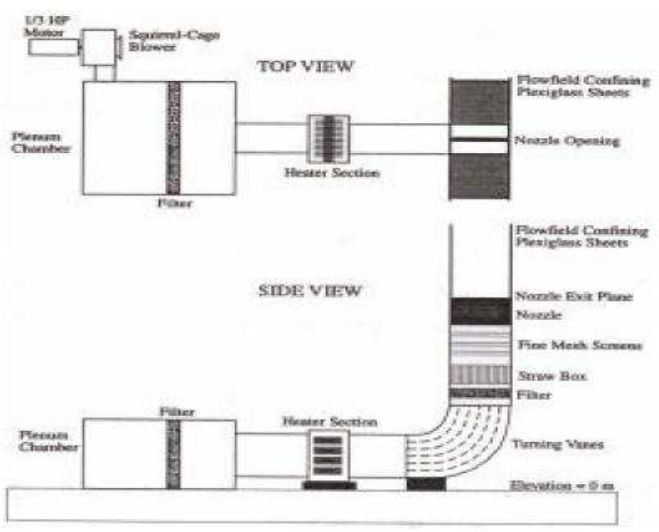

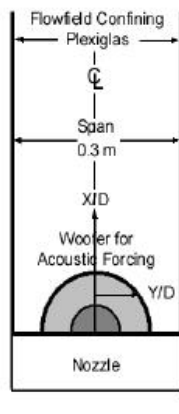

FRONT VIEW

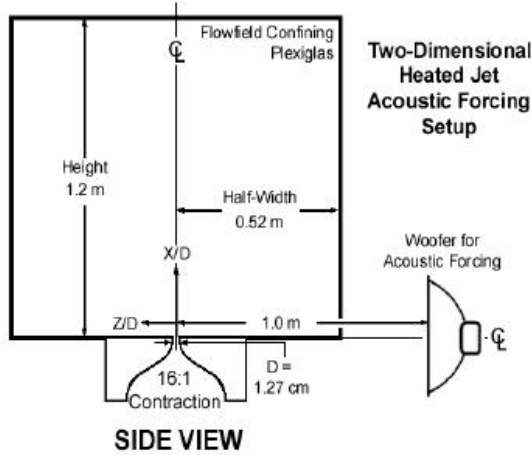

SIDE VIEW

Figure 8. 2-D heated jet facility ${ }^{15}$, left. Schematic of heated-jet exit plane, right.

The jet's response to a $240 \mathrm{~Hz}$ forcing is to regularize the jet's most-unstable Kelvin-Helmholtz instability in the jet's two bound shear layers. The first roll-up can be adjusted closer or further away from the nozzle exit plane, depending on the acoustic signal amplitude. The forcing also regularizes the first pairing, resulting in the formation 
of $120 \mathrm{~Hz}$ sub-harmonic, larger-coherence-length flow structures; however, this regularization is slightly less robust than the first roll-up. A smoke visualization of the jet and the phase-locked-averaged temperature field collected for the forced jet is shown in Fig. 9. Without acoustic forcing, the jet has a random OPD peaks pattern as a function of time, as shown in Fig. 10, left. Acoustic forcing regularizes both the amplitude and temporal frequency of the OPD pattern, as shown in Fig. 10, right. This predictable, repeatable $O P D$ pattern was used to inter-calibrate and compare the wave front sensors measurements for this study.
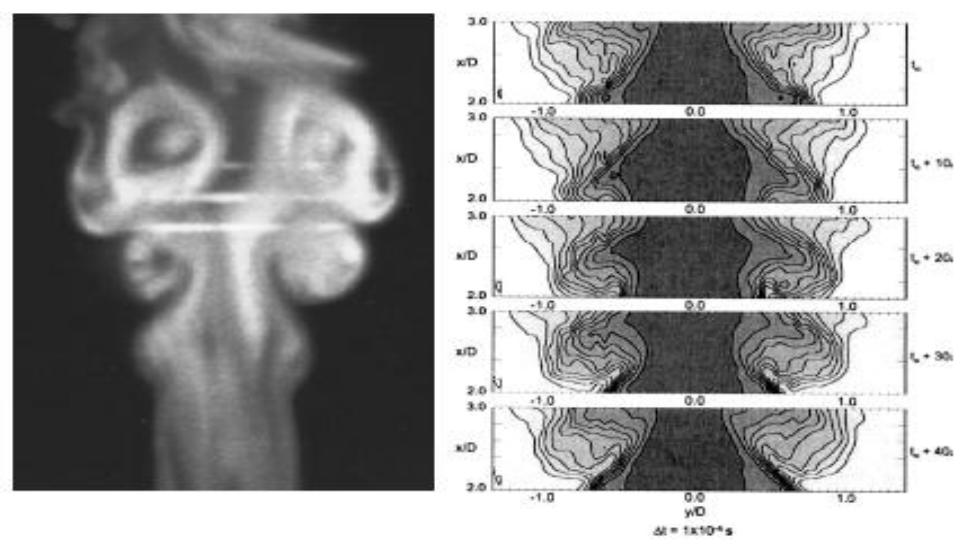

Figure 9. Smoke visualization of 2-D heated jet, left. Phase-averaged temperature profiles of the jet ${ }^{16}$, right.
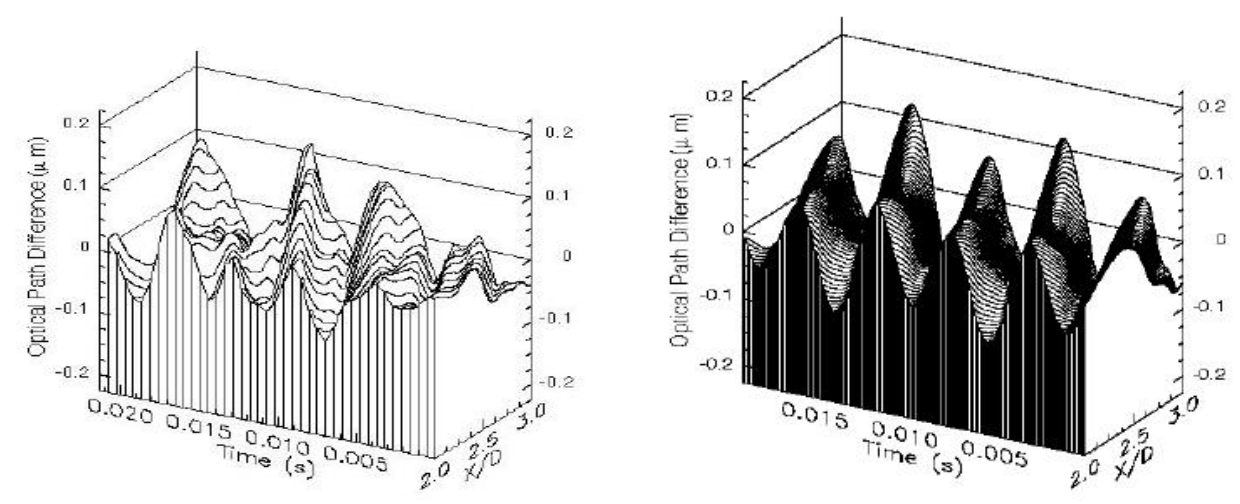

Figure 10. Time series of experimentally measured OPDs from propagation through a 2-D jet without acoustic forcing ${ }^{17}$, left. With acoustic forcing ${ }^{18}$, right.

\section{Experiment Setup}

A $2 W$ continuous Nd:YAG laser, operating at $\lambda=532 \mathrm{~nm}$ wavelength, was used to create a 1"-diameter collimated beam. The beam was directed into the heated jet facility test section using beam-steering mirrors and a beam-splitter cube, propagating the region $X / D=1 / 2 \ldots 5 / 2 ; Y / D=-1 \ldots 1$ perpendicular to the jet plane, $z$, and reflected back to the optical bench from the test section end, such that the propagating beam passes twice through the same portion of the jet, increasing the signal-to-noise ratio of the measured aberrations. The returning aberrated beam was split into two beams, the first beam was re-sized to a $\frac{1}{5}$ "-beam and passed through nutural density (ND) filters to reduce its intensity to prevent sensor saturation, and directed to the Wavefront Sciences CLAS-2D wave front sensor. The second portion of the returning beam was re-sized to a $\frac{1}{2}$ "-beam and imaged on the high bandwidth wave front sensor by passing through the re-imaging telescope. 
The jet was acoustically regularized by a woofer driven by $240 \mathrm{~Hz}$ sine waveform signal from a function generator. The speaker was located approximately $1 \mathrm{~m}$ from the jet central line (CL) as shown in Fig. 6, right. Two additional function generators were used to trigger the data acquisition systems that allowed for predetermined phase shifts with respect to forcing signal, enabling a comparison between the two wave front sensors at a series of phase shifts through the $120 \mathrm{~Hz}$ subharmonic cycle. A schematic of the optical train and electronics setup is shown in Fig. 11 .

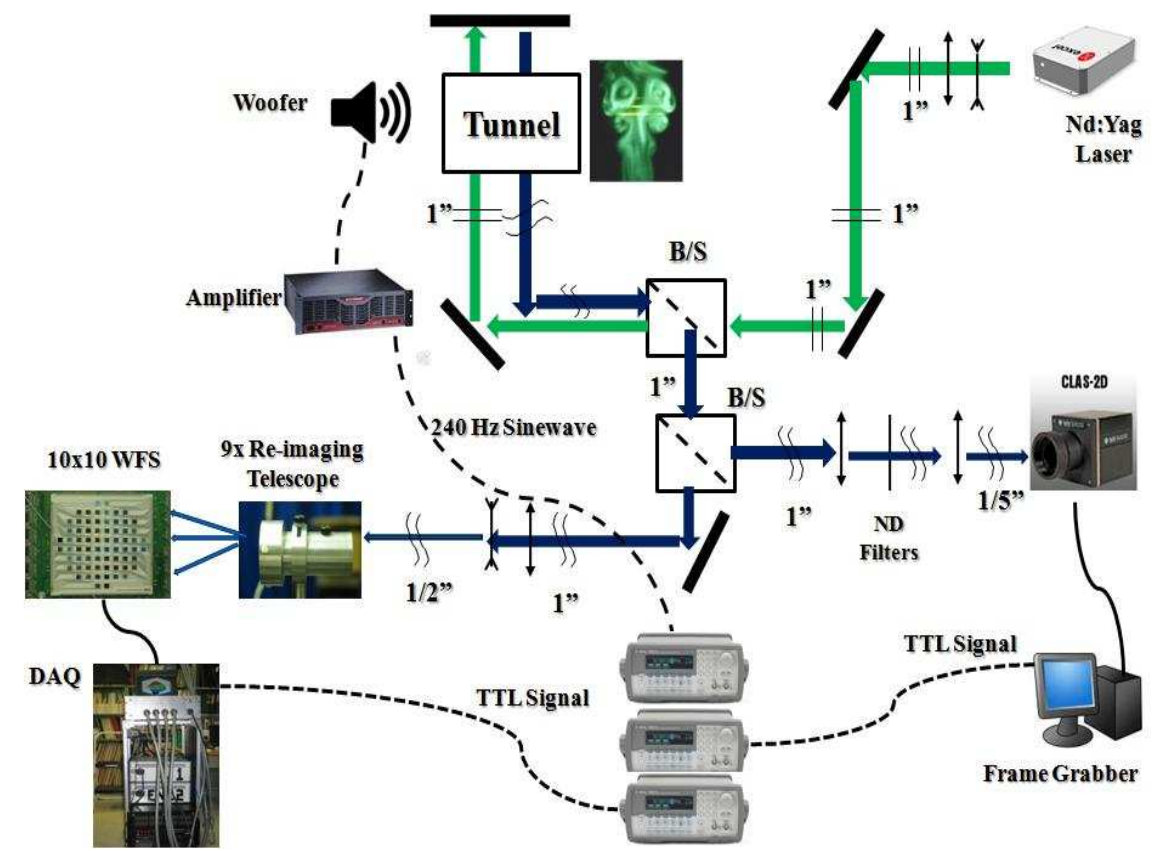

Figure 11. Scheme of optical train and electronics.

\section{Experiment Results}

\section{A. Sensor calibration and data processing}

Prior to each data run, the $10 \times 10$ sensor was calibrated by shifting the entire sensor board through a series of $x$ and $y$ positions which allowed the $x$ and $y$ voltages to be correlated with the focal spot shifts on each PSD sensor. The detector board was mounted on a two-dimensional $X Y$ linear translation stage and moved over a pre-defined two-dimensional displacement grid with respect to the light spots; each sensor was represented by a $2 x 2$ matrix, relating the two position voltages of each detector, DivX and DivY, to the two-dimensional $(x, y)$ spot position,

$$
\left[\begin{array}{l}
x \\
y
\end{array}\right]=\left[\begin{array}{ll}
K_{x X} & K_{x Y} \\
K_{y X} & K_{y Y}
\end{array}\right] \cdot\left[\begin{array}{l}
\operatorname{Div} X \\
\operatorname{Div} Y
\end{array}\right]
$$

The matrices were saved and used to reduce the four acquired voltage reading signals out of each detector into a spot displacement, $\epsilon$. Due to the photodiodes characteristics, the spots displacement-voltage relation was linear; this produced a matrix in which the diagonal elements, $K_{x X}$ and $K_{y Y}$, were much larger than the off-diagonal elements, $K_{x Y}$ and $K_{y X}$.

The analog voltage signals were digitalized and sampled in digital form at bandwidth up to $104 \mathrm{kHz}$ limited only by the data acquisition system, these position signals were then post-processed for local slopes.

These slopes were then used to construct instantaneous wave fronts based on the algorithm presented in section (3). The reconstructed wave fronts were plotted and instantaneous $O P D_{r m s}$ and $O P D_{p-p}$ values were calculated, characterizing the deviation from a perfectly collimated wave front phase. 


\section{B. Baseline Flow}

The high bandwidth wave front sensor was tested in the University of Notre Dame forced heated jet baseline facility, a well documented aero-optical environment ${ }^{15-17}$, in order to compare it to other sensor. Time-resolved raw voltages signals from the $10 \times 10$ sensor were sampled at $24 \mathrm{kHz}$. Four instantaneous wave fronts realizations of two-dimensional wave fronts are shown on Fig. 12; the convective nature of the regularized aberration is well demonstrated by the structure propagating through the beam aperture. Further, the presumption that the jet is essentially two-dimensional ${ }^{8}$, due to the acoustically forcing, is validated by noticing that the wave front profile in the streamwise direction, $x$, is the same at all spanwise directions, $y$. The time-resolved wave fronts give further insight into the periodic and growing nature of the aberrating flow field. Four typical instantaneous side-view wave fronts realizations are presented in Fig. 13. An initial aberration structure roll-up is clearly shown to start developing around $X / D=1$ with an approximate wave front amplitude of $0.1 \mu \mathrm{m}$, Fig. $13(\mathrm{~b})$, reaching a maximum aberration amplitude of approximately $0.2 \mu \mathrm{m}$ around $X / D=2$, Fig. 13 (c), and starts breaking down thereafter, Fig. 14 (d). This is supported by typical $O P D$ amplitude patterns as a function of time at four streamwise positions, $\mathrm{X} / \mathrm{D}=$ 0.7, 0.9 1.9, 2.3, shown in Fig. 14, where the periodic amplitude pattern and growing aberration nature, as a function of streamwise position, $x$, is clearly seen. Mean $O P D_{r m s}$ and $O P D_{p-p}$ values were $0.059 \mu \mathrm{m}$ and $0.25 \mu \mathrm{m}$, respectively.

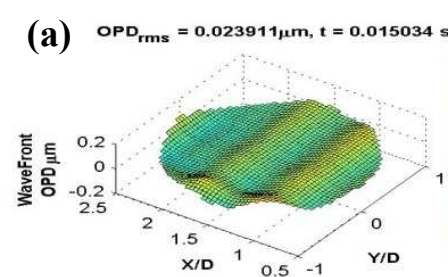

(c)

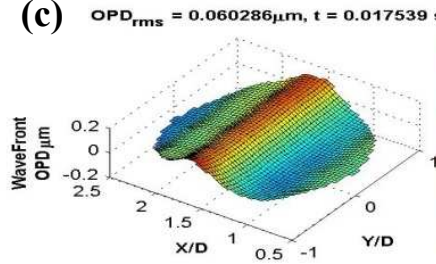

(b) $\mathrm{OPD}_{\mathrm{rms}}=0.046764 \mu \mathrm{m}, \mathrm{t}=0.016286 \mathrm{~s}$

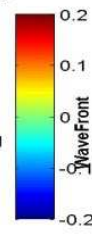

(d)
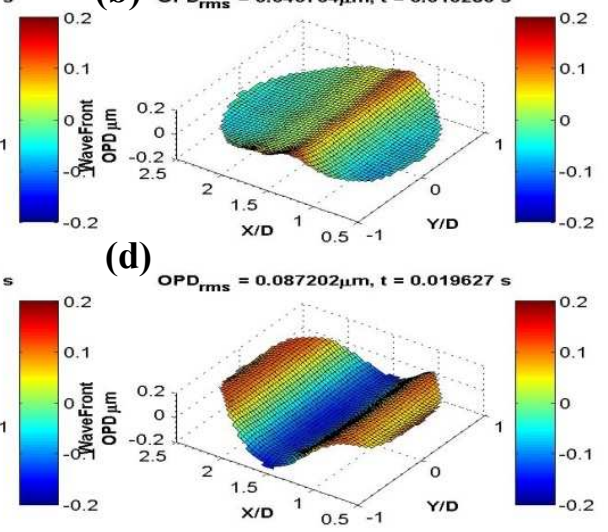

Figure 12. Typical instantaneous wave fronts realizations (2D-view) obtained with the high-bandwidth wave front sensor for a wave propagating in $X$ straemwise direction; $(1 / 2<X / D<5 / 2 ;-1<Y / D<1)$.

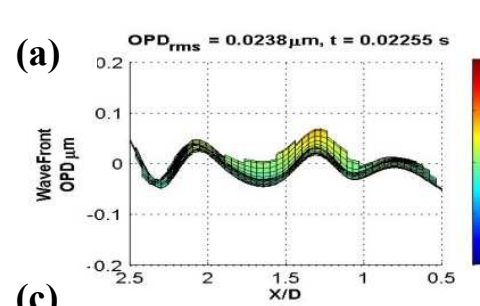

(c)

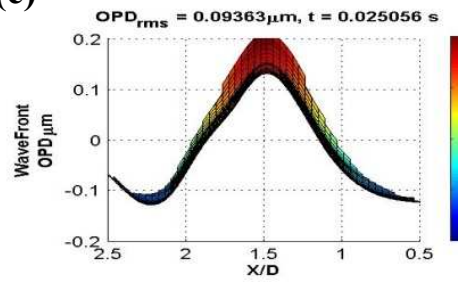

(b) $\mathrm{OPD}_{\mathrm{rms}}=0.050627 \mathrm{\mu m}, \mathrm{t}=0.023803 \mathrm{~s}$

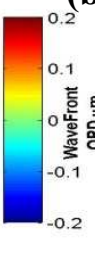

(d)

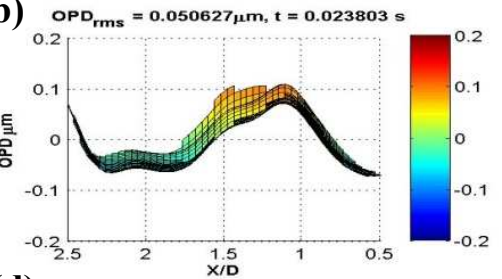

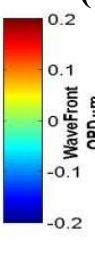

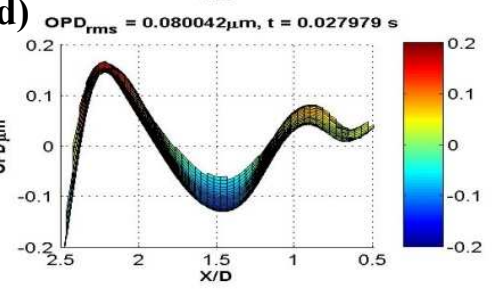

Figure 13. Typical instantaneous wave fronts realizations (Side-view) obtained with the high-bandwidth wave front sensor for a wave propagating in $X$ straemwise direction; $(1 / 2<X / D<5 / 2)$. 

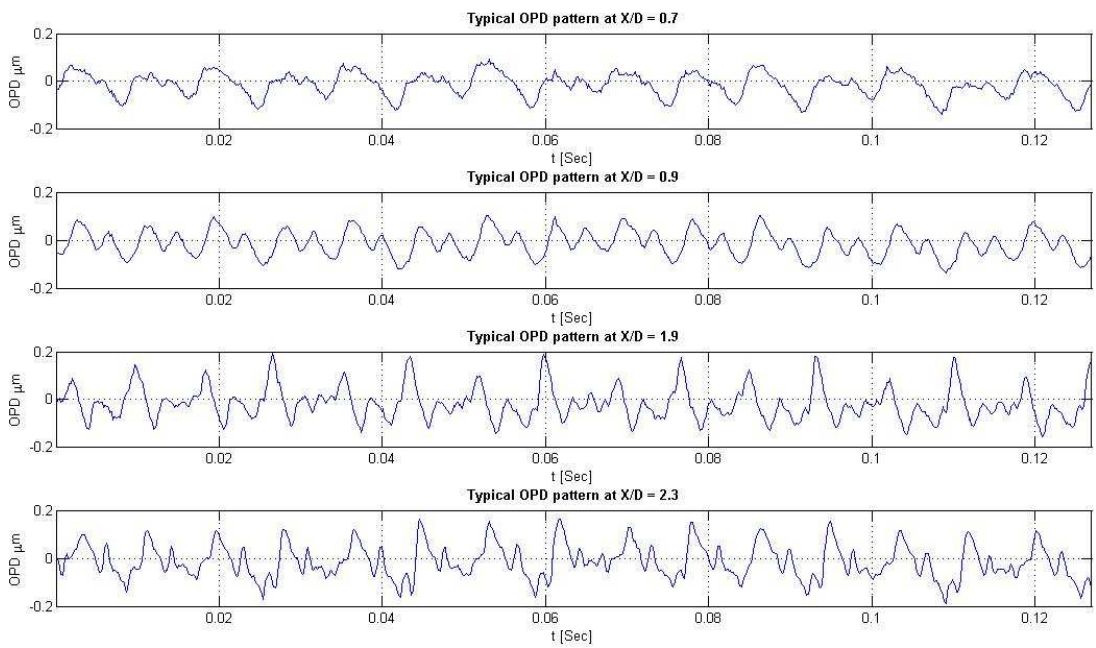

Figure 14. Typical OPD patterns at 4 streamwise positions: $X / D=0.7,0.91 .9,2.3$

\section{Comparison of wave front sensors}

In order to both validate and test the spatial resolution of the high bandwidth wave front sensor, wave fronts from the $10 \times 10$ sensor were compared to those from another sensor. The $10 \times 10$ sensor was synchronized with the Wavefront Sciences CLAS-2D wave front sensor, which although having a slower capturing rate, has higher spatial resolution than the $10 \times 10$ sensor. Both sensors were triggered to sample at $20 \mathrm{~Hz}$.

Due to the periodic nature of the acoustically forced heated jet, a phase-locked averaging was preformed to compare the two sensors. Phase-lock averaging over 400 cycles was preformed for 12 phase angles with separation between angles of $30^{\circ}$. In order to compare the two wave front sensors, a one-dimensional slice in the central location of the two-dimensional wave fronts was made. A comparison between the two sensors for the selected phase angles is presented in Fig. 15. The beam's aperture was clipped by $1.6 \mathrm{~mm}$ at the upstream edge to remove errors due to a drop in beam intensity at that edge, $0.625<\mathrm{X} / \mathrm{D}<2.5$.

The comparison between the two wave front sensors reveals that, although the high bandwidth wave front sensor has only a modest spatial resolution of $10 \times 10$ sub-apertures, it captured and reconstructed all the essential features of the two-dimensional convecting aberration. Further, despite the small peak-to-peak aberration amplitude, an average of $O P D_{p-p}=0.11 \mu \mathrm{m}$ for both sensors, the high bandwidth sensor was able to resolve the peak-to-peak aberration amplitude correctly when compared with the higher spatial resolution $C L A S-2 D$ wave front sensor.

Wave front slices from the high bandwidth wave front sensor were fit with a cubic polynomial to compare them with the wave fronts slices acquired by the $C L A S-2 D$ wave front sensor, for each phase angle a spatial matching error was calculated based on the equation

$$
\text { Error }=\sqrt{\frac{\sum_{i=0}^{N}\left[O P D_{10 \times 10}^{i}-O P D_{C L A S-2 D}^{i}\right]^{2}}{N}}
$$

where $N$ is the number of spatial points in the spanwise direction $x$. The calculated error for each phase is presented in Fig. 15. A mean error of $0.0176 \mu \mathrm{m}$ was calculated for these 12 phase angles, this value is within $20 \%$ of the mean $O P D_{p-p}$ of both wave front sensors.

Figures 16 and 17 show selected surface plots for the two sensors, these wave fronts are typical of the entire data set. A spatial $O P D_{r m s}$ value was calculated for each phase angle and shown in the plots.

Based on the two-dimensional form of Eq. (19), a mean error of $0.0223 \mu \mathrm{m}$ was calculated for the 12 acquired phase angles, this value is within $18.5 \%$ of the mean $O P D_{p-p}$ value of the wave fronts acquired with $C L A S-2 D$ wave front sensor. Mean $O P D_{r m s}$ values for the $10 \times 10$ wave front sensor and the $C L A S-2 D$ were $0.0279 \mu m$ and $0.0298 \mu \mathrm{m}$, respectively. Mean $O P D_{p-p}$ values for the $10 \times 10$ wave front sensor and the $C L A S-2 D$ were $0.1202 \mu \mathrm{m}$ and $0.1282 \mu \mathrm{m}$, respectively. 

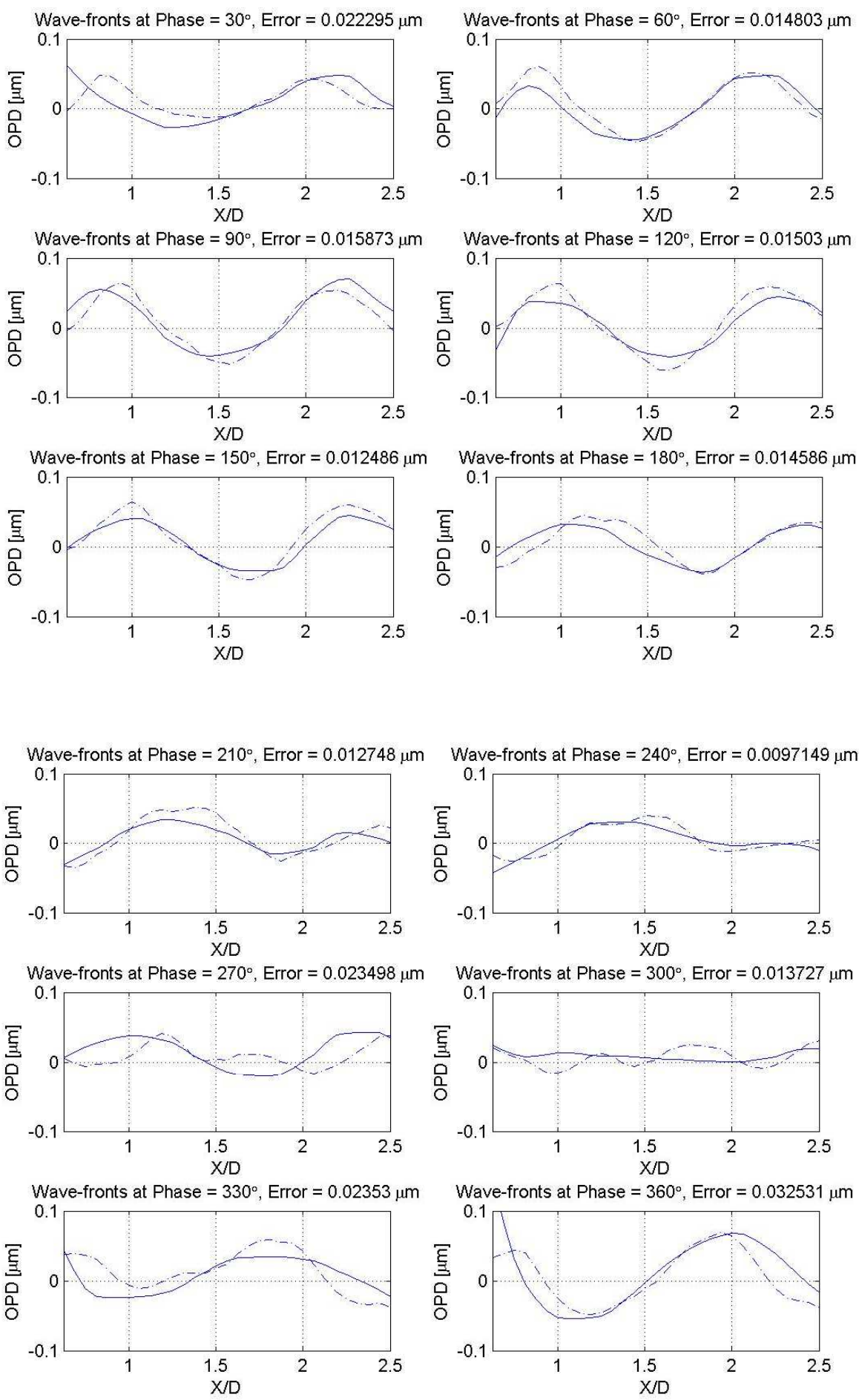

Figure 15. Comparison of phase-locked averaged wave front measurements: High-bandwidth sensor (solid line), CLAS-2D sensor (dash line); $(0.625<\boldsymbol{X} / \boldsymbol{D}<2.5)$. 

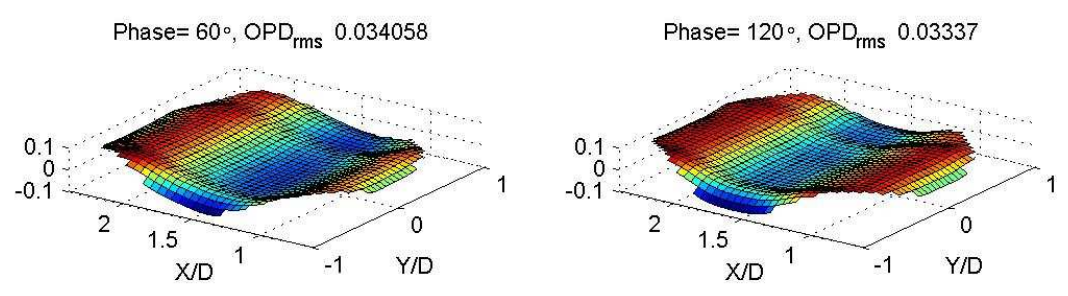

Phase $=180^{\circ}, \mathrm{OPD}_{\mathrm{rms}} 0.025593$

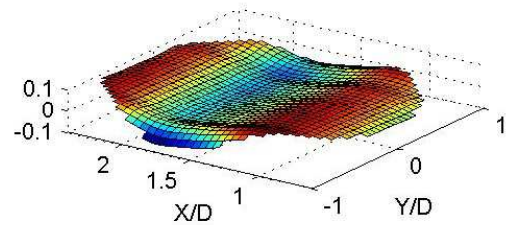

Phase $=240^{\circ}, \mathrm{OPD}_{\text {rms }} 0.018037$

Phase $=300^{\circ}, \mathrm{OPD}_{\text {rms }} 0.010855$
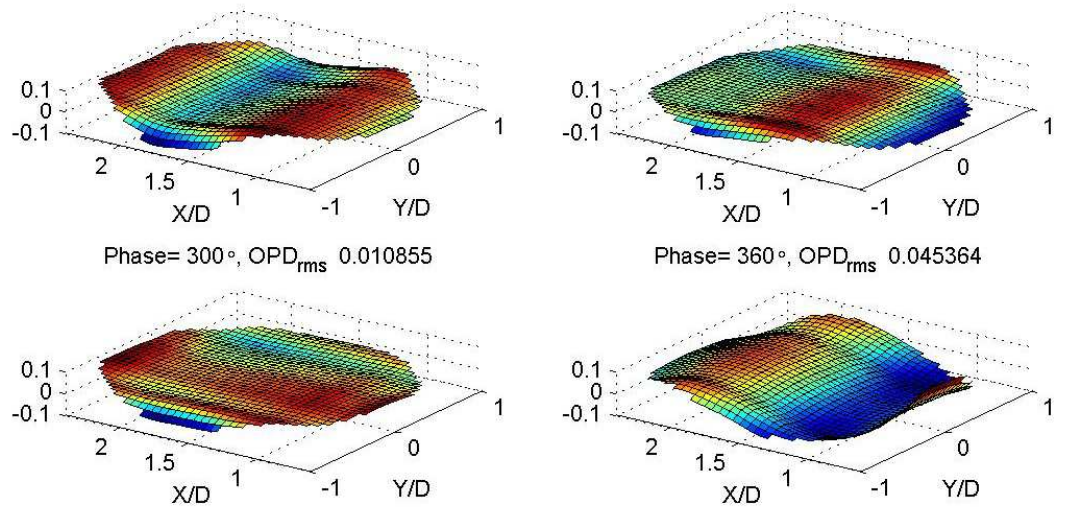

Figure 16. Typical instantaneous phase-locked averaged wave fronts realization obtained with the high bandwidth wave front sensor at various phase angles; $\left(1 / 2<X /{ }_{D}<5 / 2 ;-1<Y / D<1\right.$; Scale in $\left.\mu m\right)$.

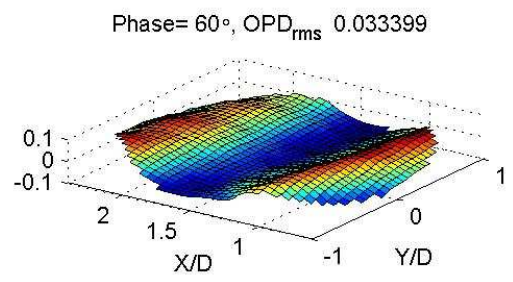

Phase $=180^{\circ}, \mathrm{OPD}_{\text {rms }} 0.030614$

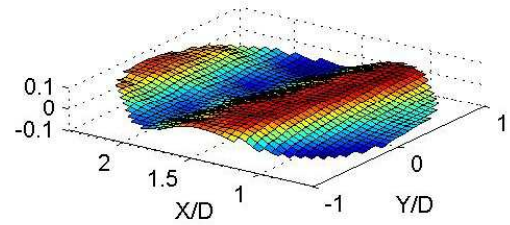

Phase $=300^{\circ}, \mathrm{OPD}_{\mathrm{rms}} 0.013801$

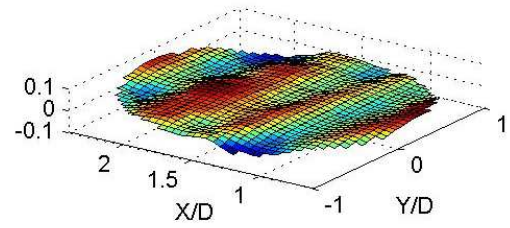

Phase $=120^{\circ}, \mathrm{OPD}_{\mathrm{rms}} 0.041178$

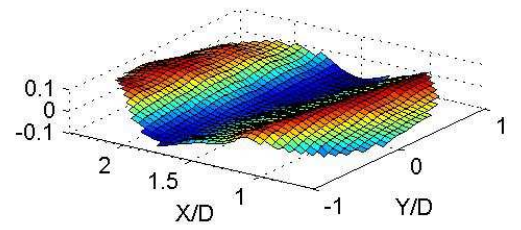

Phase $=240^{\circ}, \mathrm{OPD}_{\text {rms }} 0.023831$

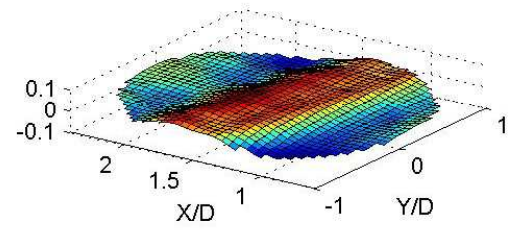

Phase $=360^{\circ}, \mathrm{OPD}_{\mathrm{rms}} 0.035865$

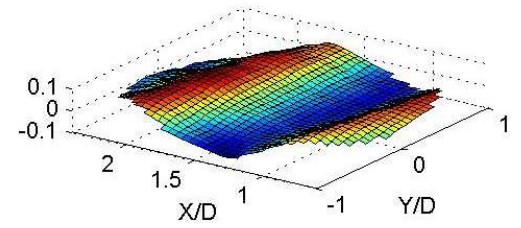

Figure 17. Typical instantaneous phase-locked averaged wave fronts realization obtained with $C L A S$ - $2 D$ wave front sensor at various phase angles; $(1 / 2<X / D<5 / 2 ;-1<Y / D<1$; Scale in $\mu m)$. 


\section{Conclusion}

An analog high bandwidth, two-dimensional, wave front sensor was developed and tested against a commercially available CLAS-2D Wavefront Sciences wave front sensor. The analog nature of the new sensor enables a high sampling rate for aero-optical convecting aberrations, capable of capture-rate greater than $100 \mathrm{kHz}$, which is essential for investigating aero-optical effects and developing adaptive optics systems that can mitigate high content temporal bandwidth frequencies.

The spatial and temporal resolutions of the new sensor were tested on an acoustically forced heated jet facility and compared to the $C L A S-2 D$ wave front sensor, which has a higher spatial resolution of $33 \times 44$ sub-aperture, than the $10 \times 10$ sub-apertures resolution that the high-bandwidth sensor has. It was shown that despite the modest spatial resolution, the new sensor was capable of resolving the essential spatial character and demonstrating a convective nature of the aberration.

The new sensor can be used to investigate a large number of aero-optical effects, which could not be addressed before due to the limited sampling rate capability of the current available wave front sensors.

\section{Acknowledgments}

These efforts were sponsored by the Office of Naval Research, ONR under Grant Number N00014-07-1-0291. The U.S. Government is authorized to reproduce and distribute reprints for governmental purposes notwithstanding any copyright notation thereon.

\section{References}

${ }^{1}$ Jumper, E. J., and Fitzgerald, E. J., "Recent Advances in Aero-Optics," Progress in Aerospace Sciences, Vol. 37, 2001, pp. 299-339.

${ }^{2}$ Fitzgerald, E. J. and Jumper E. J., "The optical distortion mechanism in a nearly incompressible free shear layer," Journal of Fluid Mechanics, Vol. 512, 2004, pp. 153-189.

${ }^{3}$ Nightingale, S.Gordeyev, E. Jumper, B. Goodwine and J. Siegenthaler, "Regularizing Shear Layer for Adaptive Optics Control Applications", 36th AIAA Plasmadynamics and Laser Conference, Toronto, Canada, June, 2005.

${ }^{4}$ Tyson, R.K., Principles of Adaptive Optics, Academic Press, Inc., San Diego, 1991.

${ }^{5}$ Air Force Office of Scientific Research, AFOSR, under Grant Number, F49620-02-C-0031

${ }^{6}$ Malacara D., Optical Shop Testing, Wiley, 1978.

${ }^{7}$ Kelin M.V., Optics, Wiley, New York, 1970.

${ }^{8}$ Wyckham C., Zaidi S., Miles R., and Smits A., "Measurement of Aero- Optic Distortion in Transonic and Hypersonic, Turbulent Boundary Layers with Gas Injection,", AIAA Paper 2005-4755, Toronto, Ontario, June 2005

${ }^{9}$ Hugo, R. J., and Jumper, E. J., "Quantification of Aero-Optical Phase Distortion Using the Small-Aperture Beam Technique," AIAA Journal, Vol. 33, No. 11, 1995, pp. 2151-2157.

${ }^{10}$ Gordeyev S., Duffin D., and Jumper E. J., "Aero-Optical Measurements Using Malley Probe and High-Bandwidth 2-D Wavefront Sensor", International Conference on Advanced Optical Diagnostics in Fluids, Solids and Combustion, Tokyo, Japan, Dec, 2004.

${ }^{11}$ Duffin, D., Gordeyev, S. and Jumper, E. J., "Comparison of Wavefront Measurement Techniques on a Two-Dimensional Heated Jet", AIAA Paper 2004-2406, Portland, June 2004.

${ }^{12}$ Fried D. L., "Least-square fitting a wave front distortion estimate to an array of phase-difference measurements," Journal of Optical Society of America, 67, 1977, 370-375.

${ }^{13}$ Hudgin $R$. H., "Wave front reconstruction for compensated imaging," Journal of Optical Society of America, 67, 1977, 375378.

${ }^{14}$ Southwell W. H., "Wave front estimation from wave front slope measurements," Journal of Optical Society of America, 70 , 1980, 998-1006.

${ }^{15}$ Hugo, R. J., "Quantifying the Spatio-Temporal Effects of Optically-Active Turbulent Flow fields on a Coherent Optical Wave," Ph.D. Dissertation, Department of Aerospace and Mechanical Engineering, University of Notre Dame, Notre Dame, IN, 1995.

${ }^{16}$ Hugo, R. J., and Jumper, E. J., "Implications of the Homogeneous Turbulence Assumption on the Aero-Optic Linking Equation," Optical Techniques in Fluid, Thermal, and Combustion Flow, Vol. 2546, edited by S.S. Cha and J.D Trollinger, SPIE-International Society of Optical Engineering, Bellingham, Washington, USA, 1995, pp. 189-200.

${ }^{17}$ Hugo, R. J., and Jumper, E. J., "Experimental Measurement of a Time-Varying Optical Path Difference by the SmallAperture Beam Technique,” Applied Optics, Vol. 35, No. 22, Aug. 1996, pp. 4436.

${ }^{18}$ Hugo, R. J., and Jumper, E. J., "Constant Current Anemometry and its impact on Aero-Optical Measurements," AIAA Paper 95-1986, Jun. 1995. 